\title{
Period-doubling density waves in a chain
}

\author{
Joost H J van Opheusden and Theo P Valkering \\ Center for Theoretical Physics, University of Twente, PO Box 217, 7500AE Enschede, \\ The Netherlands
}

Received 25 April 1988

Accepted by D K Campbell

\begin{abstract}
We consider a one-dimensional chain of $N+2$ identical particles with nearest-neighbour Lennard-Jones interaction and uniform friction. The chain is driven by a prescribed periodic motion of one end particle, with frequency $v$ and 'strength' parameter $\alpha$. The other end particle is held fixed.

We demonstrate numerically that there is a region in the $\alpha-v$ plane where the chain has a stable state in which a density wave runs to and fro between the two ends of the chain, similarly to a ball bouncing between two walls. More importantly, we observe a period-doubling transition to chaos, for fixed $v$ and increasing $\alpha$, while the localised (solitary wave) character of the motion is preserved.

Calculation of the Floquet multipliers along the bifurcating branch shows that the chain behaves as a system of one dominant nonlinear oscillator, which is weakly coupled to $N-1$ linear oscillators. The former is responsible for the period-doubling cascade, whereas the latter only 'follow' without disturbing this process.

These phenomena can be understood as follows. The chain without friction and driving has a family of solutions parametrised with the energy, representing the bouncing density wave. This family will be identified as the dominant oscillator. In the driven and damped system the trajectory remains in the neighbourhood of this family. We demonstrate how to choose coordinates which separate the dominant oscillator from the 'irrelevant' linear oscillators. In this way a model is formulated which clarifies the effectively one-degree-of-freedom behaviour of the chain.
\end{abstract}

PACS numbers: $0320,0340 \mathrm{~K}, 6320 \mathrm{P}$

\section{Introduction}

In this paper we consider a one-dimensional system of identical particles (figure $1(a)$ ), with nearest-neighbour interaction of the 6-12 Lennard-Jones type, which has the form as sketched in figure $1(b)$. On each particle in the chain acts a friction force proportional to its momentum, and the friction coefficient is equal for all particles. One end is kept fixed, while the chain is forced externally by a prescribed motion of the opposite end particle, parallel to the chain. This motion is periodic and has the shape as given in figure 2. It depends on two parameters: the frequency $v$, and a strength parameter $\alpha$, which is the second derivative of the motion at its minimum.

After some trial and error we obtain a set of values for $\alpha$ and $v$, such that the 

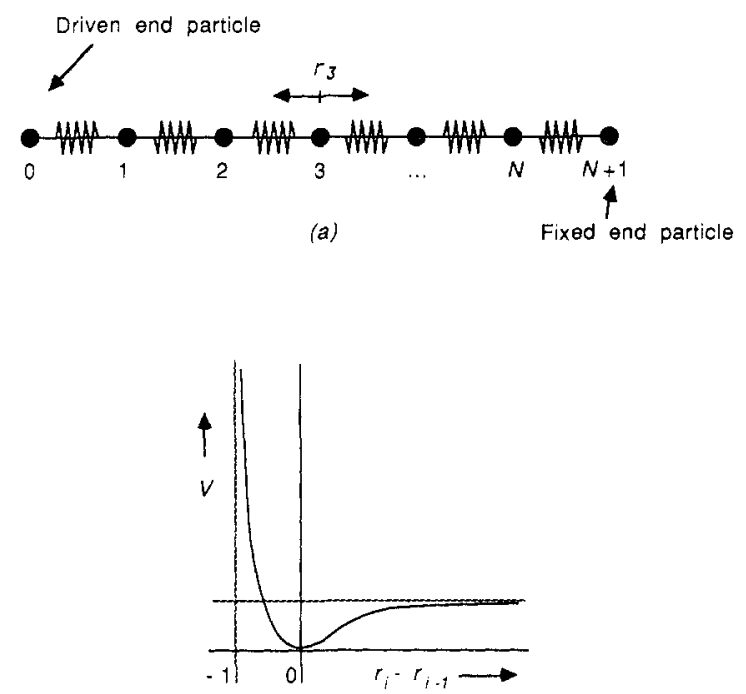

(b)

Figure 1. The system we investigate consists of a one-dimensional chain of point particles with uniform friction. (a) One end of the chain is held fixed, while the other end is moved periodically in a specific way. The particles interact with their nearest neighbours through a 6-12 Lennard-Jones potential, sketched in (b).

chain has a stable state in which a sharp density wave runs back and forth between the two ends of the chain, with the period of the driving term. More importantly, we observe period-doubling sequences, for fixed $v$, and increasing $\alpha$, where the localised solitary wave character of the state is preserved. This phenomenon is similar to what is found for a ball bouncing between a fixed and a periodically moving wall. Obviously, in the latter case, only one degree of freedom is involved. The aim of the paper is to discuss in what sense the motion of the chain also is essentially a phenomenon with a single degree of freedom.

To obtain insight into this question, we calculate the Floquet multipliers of the

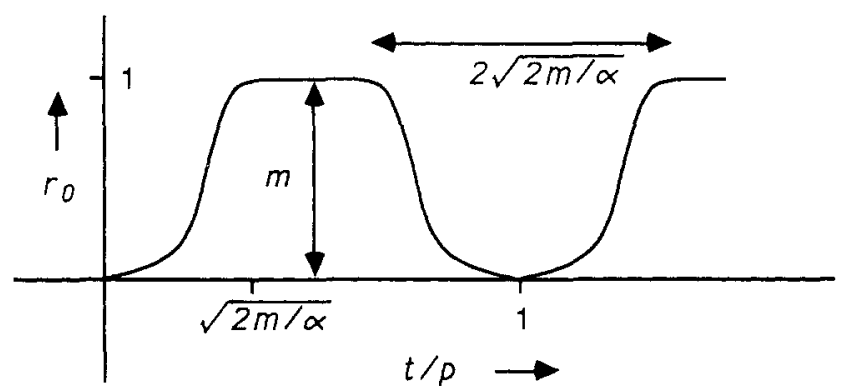

Figure 2. Motion of the driven end of the chain, $r_{0}(\tau)=\alpha \tau^{2}-\alpha^{2} \tau^{4} / 4 m$. The strength parameter $\alpha$ gives the second derivative at $\tau=0$, where the motion is quadratic in time. For $\alpha>2 m$ the quartic term gives a smooth transition to the cutoff $m$. The cutoff appears necessary, as otherwise the compression of the chain becomes so large that the period doubling process for one solitary wave is disturbed. 

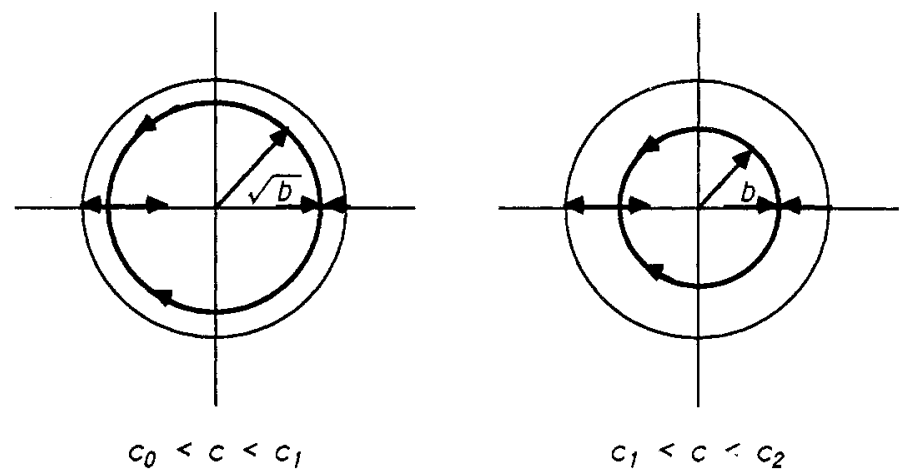

Figure 3. Period doubling for a single damped and driven anharmonic oscillator. The Floquet multipliers, the eigenvalues of the linearisation of the system around the stable periodic orbit, move over a circle in the complex plane with radius $\sqrt{b}$, with $b$ the volume contraction factor of phase space per period. The eigenvalues start on the positive real axis, and move to the negative real axis as a function of some driving parameter $c$. The period doubling takes place when one eigenvalue becomes -1 . Then the process repeats itself, the multipliers now being evaluated over two periods rather than one, until at $c=c_{2}$ the period four appears.

solution (i.e. the eigenvalues of the linearisation of the system around the periodic orbit) when it goes through the period-doubling sequence. For the bouncing ball problem these eigenvalues show simple behaviour; one pair of complex conjugate eigenvalues moves on a circle with radius $\sqrt{b}$, with $b$ the area contraction rate in phase space, towards $-\sqrt{b}$. Then they separate along the negative real axis. The period doubling takes place when one eigenvalue passes $\lambda=-1$ (figure 3). This process repeats itself for the orbit with double period; the eigenvalues starting at $\lambda=1$ and $\lambda=b^{2}$ move along a circle with radius $b$ to $\lambda=-1$ and $\lambda=-b^{2}$, etc.

In the case of the chain the process is necessarily more complicated, since there are $N$ pairs of eigenvalues. Related to the uniform damping, the eigenvalues of a periodic motion all appear to move on a circle in the complex plane with radius $\beta^{p / 2}$, $p$ being the period and $\beta^{N}$ now being the volume contraction rate per unit time in the $2 N$-dimensional phase space. The formalism and the properties of the multipliers for the system we are considering are described in detail in $\$ 2$. Because all multipliers move on the same circle the pair of eigenvalues associated with the period doubling necessarily collides with the others during the process of the period-doubling cascade.

Section 3 describes the observations we have made for a number of computer experiments of the system. An appendix describes the discrete-time dynamics of the chain, which we have used in these computer simulations. The main result of these experiments, in which we are dealing with a system having many degrees of freedom $(N=5)$, is that the observed eigenvalue behaviour is very similar to that of one dominant oscillator, which is only weakly coupled to $N-1$ other oscillators. The former is responsible for the period doublings, whereas the latter only follow without disturbing the process.

This phenomenon can be understood as follows. In the conservative chain, without friction and driving, there is a family of periodic solutions, representing the undisturbed bouncing density wave. The energy can serve as the describing parameter, while the frequency and the shape of the wave depend on the energy. 
Now suppose, as suggested by the experimental evidence, that in the driven and damped system the trajectory remains in the neighbourhood of this family. More precisely, if an orbit of the conservative chain is represented by $v(E, \varphi), \varphi=\omega(E) t$, then an orbit of the damped and driven system is given by $v(E(t), \varphi(t))$, plus a small term. In $\$ 4$ we demonstrate how with this family one may define coordinates, suitable for the description of the dynamics of $E(t) \varphi(t)$ and $\zeta(t)$, the small term. Thus one obtains a more quantitative understanding of the essentially one-degreeof-freedom character of the phenomena as observed.

Temporally chaotic phenomena related to spatially coherent structures occur in many physical systems, and in a variety of forms (cf for instance [1]). An extensively investigated example is that of a solitary wave in an optical bistable ring cavity [2]. More closely related to our work are the numerical studies of the Toda chain [3], and the (continuous) sine-Gordon system [4]. In these papers one usually excites the system with a simple sinusoidal driving term, and one finds a diversity of chaotic phenomena for increasing amplitude. In some cases low-dimensional chaotic behaviour is found, related to soliton or kink modes of the system. The reason we have chosen a different driving term is that we want to excite precisely that single nonlinear mode in the non-integrable system, the solitary wave, and observe its period-doubling transition to chaos.

\section{Equations of motion}

The equations of motion are formulated. It is shown that, for uniform friction, the flow in phase space is symplectic in a generalised way, so that the Floquet multipliers satisfy well known symmetry relations.

If the time $t$ is scaled with the period $1 / v$ the equations of motion of the chain are

$$
r^{\prime \prime}=-v^{-2} \operatorname{grad} U(r ; \tau)-\gamma r^{\prime}
$$

Here $r$ is an element of $R^{N}$ representing the deviations from the equilibrium positions of the particles, which depends on the scaled time $\tau=v t$, and $\gamma$ is the friction coefficient divided by the frequency. The potential $U$ has the form

$$
U(r ; \tau, \alpha)=\sum_{i} V\left(r_{i}-r_{i-1}\right) \quad r_{0}=r_{0}(\tau ; \alpha) \quad r_{N+1}=0 .
$$

For the interaction $V$ we choose the Lennard-Jones potential

$$
V(x)=(x+1)^{-12}-2(x+1)^{-6} \text {. }
$$

The prescribed motion of the zeroth particle has unit period and is the periodic continuation of a function defined on the interval $-\frac{1}{2} \leqslant \tau \leqslant \frac{1}{2}$ :

$$
\begin{aligned}
& r_{0}(\tau, \alpha)=\left\{\begin{array}{llc}
\alpha \tau^{2}-\alpha^{2} \tau^{4} / 4 m & \text { if } & |\tau| \leqslant \sqrt{2 m / \alpha} \\
m & \text { if } & \sqrt{2 m / \alpha} \leqslant|\tau| \leqslant \frac{1}{2}
\end{array}\right. \\
& r_{0}(\tau+1, \alpha)=r_{0}(\tau, \alpha)
\end{aligned}
$$

where $m$ is a constant which is kept equal to unity in our calculations. Observe that the frequency $v$ in the equations of motion appears as a prefactor of the potential.

The second-order differential equation $(2.1)$ is readily transformed to a firstorder system

$$
p^{\prime}=-v^{-1} \operatorname{grad} U(r ; \tau)-\gamma p \quad r^{\prime}=p v^{-1}
$$


Since the divergence of the right-hand side equals $-N \gamma$, this flow reduces volume in phase space with a constant rate: a volume decays with $\exp (-N \gamma \tau)$.

If $w(\tau)=\left(p_{1}, r_{1}, p_{2}, \ldots\right)(\tau)$ denotes an orbit of (2.2), the 'time one' map $F$ is defined in the usual way:

$$
w(1)=F(w(0))
$$

Since the flow reduces volume at a constant rate, this nonlinear map has constant Jacobian, i.e. the derivative $\mathbf{d} F$ satisfies

$$
\operatorname{det} \mathbf{d} F=\mathrm{e}^{-\gamma N} \text {. }
$$

To obtain a second, and for our problem very relevant, property of $F$ observe that equations (2.2) follow from variation of the functional

$$
A=\int \mathrm{d} \tau \mathrm{e}^{\gamma \tau}\left[\left(p, r^{\prime}\right) / 2-v^{-1} H(p, r ; \tau, \alpha)\right] \quad H=(p, p) / 2-U
$$

with (, ) denoting the usual inner product in $R^{N}$. As a result the flow is symplectic in a generalised way, i.e.

$$
\mathbf{d} F^{T} J \mathbf{d} F=\mathrm{e}^{-\gamma} J \quad J_{0}=\left[\begin{array}{rr}
0 & 1 \\
-1 & 0
\end{array}\right] \quad J=J_{0} \oplus J_{0} \oplus \ldots \oplus J_{0} .
$$

On the basis of this relation one readily derives that if $\mu$ is an eigenvalue of $\mathbf{d} F$, the same holds for

$$
\mu^{*}, \beta \mu^{-1} \text { and } \beta \mu^{-1 *} \quad \text { with } \beta=\mathrm{e}^{-\gamma} .
$$

This is a generalisation of the well known result for symplectic matrices (cf [5]). It is easily proved as follows: $\mu^{*}$ is an eigenvalue since $\mathbf{d} F$ is real. If $z$ is an eigenvector corresponding to $\mu$ then (2.6) yields immediately $\mathbf{d} F^{\mathrm{T}} J z=\mu^{-1} \beta J z$, so that $\mu^{-1} \beta$ is an eigenvalue of $\mathbf{d} F^{\mathrm{T}}$ and thus of $\mathbf{d} F$.

A consequence is that the eigenvalues always occur in pairs or in quadruples, which are symmetric with respect to the circle with radius $\beta^{1 / 2}$ in the complex plane. Particularly relevant is that an eigenvalue can move off or arrive on the ' $\beta^{1 / 2}$ circle' only if it coincides with another one.

\section{Numerical observations}

In order to be able to numerically integrate the equations of motion, we have, of course, to use some discretisation of the time. The transition from a continuous-time to a discrete-time system and the resulting set of equations is described in the appendix. Essentially the discretisation involves calculating the forces only at small time intervals $\Delta t$, and keeping them constant between time steps.

In our calculations we have studied a chain of five particles $(N=5)$. The value of the cutoff parameter $m$ (cf equation $(2.1 d)$ has always been unity $(m=1)$, and the time step has been kept constant at $\Delta t=v^{-1} / 400$, which is small enough to give the proper behaviour at collisions between two beads of the chain. Note that in the continuous system there is an infinite potential barrier preventing beads from 'passing through each other'. In the discrete system this sort of behaviour is not excluded, but for the value of $\Delta t$ we have chosen it did not occur. The value of the dimensionless friction constant has been kept at $\gamma=0.3$. 
For three different driving periods we considered a stable equilibrium state with the same period as the driving force, i.e. period one with respect to the reduced time $\tau$. For each case we investigated the destabilisation behaviour that occurs when we change $\alpha$. The computer program has the possibility of gradually changing the strength parameter $\alpha$, and also the frequency $v$, during the simulation. In the actual implementation, instead of adjusting $v$, we change the strength of the interaction potential, which according to $(2.1 a)$ is completely equivalent. This enables us to monitor the behaviour of the Floquet multipliers not only when $\alpha$ but also when $v$ is changed. If the increase occurs slowly enough, the system remains near the stable asymptotic orbit. In particular the eigenvalues of the Jacobian matrix found by integrating the linearised equations of motion along the orbit are near that of the periodic equilibrium orbit at the momentary values of $\alpha$ and $v$.

The excitation we are interested in is that of a single pulse-like wave bouncing between the driven and the fixed end (figure 4(a)). Next to the single-wave solutions, two-wave and other excitations also exist [6]. The point is to choose initial conditions inside the basin of the asymptotically stable single-wave orbit. To obtain such a state we start at an intermediate value of the driving amplitude $(\alpha \approx 0.5)$, all particles at their static equilibrium positions $\left(r_{0}, \ldots, r_{N+1}=0\right)$ and all velocities zero, except $v_{1} \simeq N v\left(v_{2}, \ldots, v_{n}=0\right)$. Note that for hard point particles one would expect $v_{1} \simeq 2 N v$; the value we obtain illustrates the finite extent of the LennardJones potential. After a while a stable solitary wave develops with a period equal to that of the driving force. Single-wave states at different driving frequencies and strength parameter are obtained by this procedure, or by slowly changing either $\alpha$ or $v$, starting from an established stable orbit.

Our first experiment was performed at $v=10$. When $\alpha$ is increased the first period-doubling bifurcation occurs at $\alpha \simeq 2.086$. In the $r-t$ plot this is apparent in the phase of the density wave. Also the time plot of the energy shows the period doubling. In the Fourier spectra of both the energy and the position of the first particle a sharp peak is found at $v=5$, i.e. half the driving frequency. All Floquet multipliers are initially on a circle with radius $\beta^{1 / 2}=\exp (-\gamma / 2)$ (cf equation (2.7)). Also we find that the phase of one pair of eigenvalues $\left(\lambda_{0}, \lambda_{0}^{*}\right)$ changes much more rapidly than that of the other four. When $\lambda_{0}$ and $\lambda_{0}^{*}$ meet at the negative real axis they leave the inner circle, and for $\lambda_{0}=-1$ a period-doubling bifurcation takes place (figure 5). If we calculate the Floquet multipliers over two periods we find all $\lambda$ inside the unit circle. Moreover we can compare the values of $\lambda_{1}, \ldots, \lambda_{4}$, the multipliers not involved in the destabilisation, shortly before and after the bifurcation, and identify pairs $\lambda_{i}(2 p) \simeq \lambda_{i}^{2}(p)$. This indicates that a slow phase change still occurs, regardless of the fact that the equilibrium orbit has altered considerably.

Increasing $\alpha$ yet further we observe a period-doubling cascade. The higher-order periods are visible as sharp peaks in the spectrum (figure $4(d)$ ). Shortly after the period 32 appears the spectral lines broaden (figure $4(f)$ ), indicating chaotic behaviour. An important observation is that the motion remains a localised density wave, running back and forth in the chain like a particle. When we follow the behaviour of the Floquet multipliers with increasing $\alpha$ we observe the following phenomena. Still one pair of eigenvalues $\left(\lambda_{0}\right)$ changes rapidly, while four other pairs $\left(\lambda_{i}\right)$ move much more slowly. The fast pair repeatedly moves over the inner circle to $\lambda_{0}=-1$, giving the consecutive period doublings. In order to do so, it has to pass the others. The behaviour of the eigenvalues when the fast one meets a slow one 

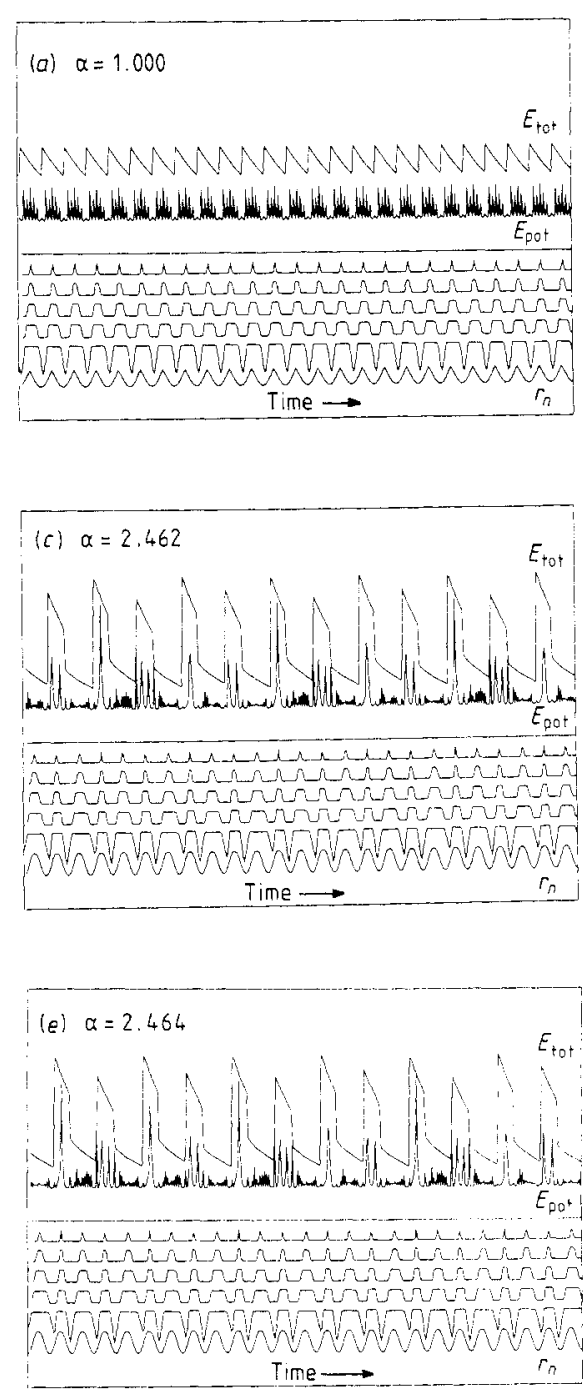
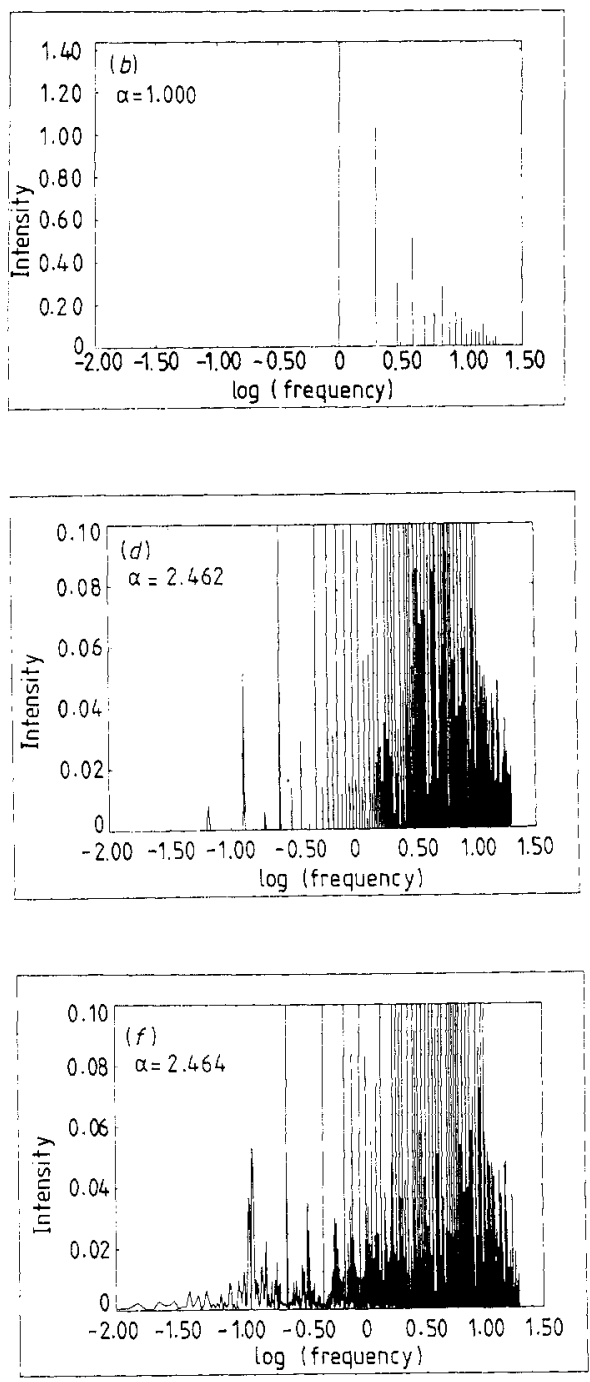

Figure 4. Motion and spectra of the chain for several values of the strength parameter. In all cases the frequency is $v=10$ and $\gamma=0.3$. In the $r-t$ plots $(a, c, e)$ we give the positions of the particles $\left(r_{n}\right.$, bottom), the potential $E_{\text {pot }}$ and the total energy $E_{\mathrm{tor}}$ as a function of time. The spectro $(b, d, f)$ are the discrete Fourier transforms of the positions of the first particle in the chain. For $\alpha=1.0$ the excitation $(a)$ is a period-one orbit, 0 localised density wave which bounces back and forth in the chain with a period equal to that of the driving term. The spectrum (b) taken over the positions of the first particle (next to the driven end particlel shows a sharp peak at the driving frequency, and of course its higher harmonics. For $\alpha=2.462$ the period-doubling cascade has developed and a period 16 is visible in the spectrum (d) as a small but sharp peak. Also periods $8, \frac{16}{3}, 4$, $\frac{16}{5}$, etc are visible as sharp peaks. Note that we have changed the scale of the vertical axis, relative to $(b)$. In the $r-t$ plot $(c)$ the period doubling is apparent in the phase of the density wave. The energy as a function of time also shows the consecutive period-two modulations. For $\alpha=2.464$, only slightly higher than for period 16 , the $r-t$ plot $(e)$ is not very different, and only in the potential energy, indicating the detailed two-particle collisions, can one see that the periodicity has disappeared. The Fourier spectrum ( $f$ ) shows the broadening of the peaks to a more continuous spectrum indicating chaotic behaviour. 

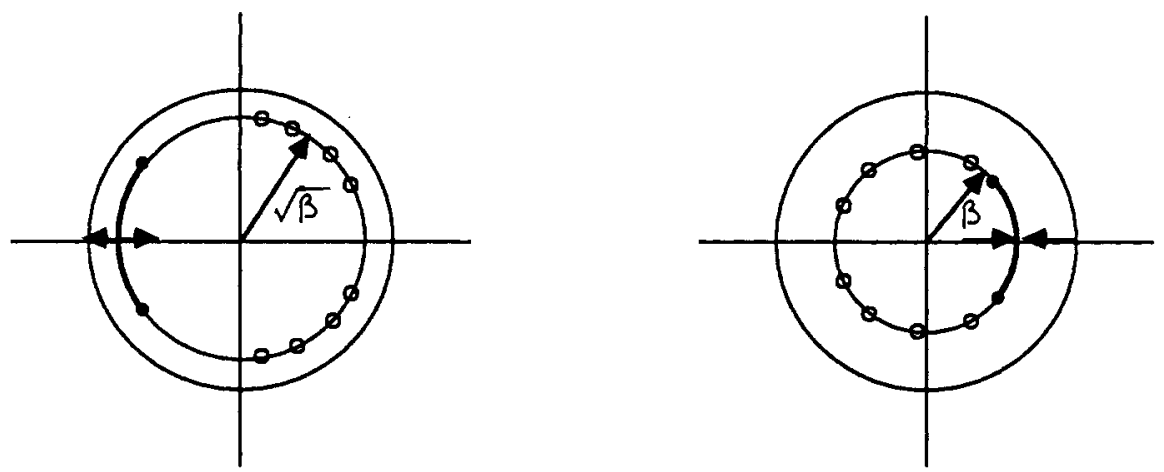

Figure 5. Behaviour of the Floquet multipliers for the chain with five particles $(N=5)$. For the stable period-one orbit, i.e. a density wave running to and fro with the period of the driving force, the eigenvalues are on a circle with radius $\sqrt{\beta}$, where now $\beta^{N}$ is the volume contraction factor per period in $2 \mathrm{~N}$-dimensional phase space. When the largest eigenvalue, which moves much faster as a function of the strength parameter $\alpha$ than the other four pairs, crosses the unit circle at -1 , the period-doubling bifurcation occurs. Here the process for going to period four is complicated because in travelling over the inner circle with radius $\beta$ the fast-moving eigenvalue catches up with four slow ones. Experimentally, however, we find that when it meets a slow one they simply pass each other without showing complicated behaviour.

strongly contrasts with what happens when two slow ones meet. When the fast eigenvalue catches up with a slow one it seems simply to pass through, at least within experimental error. The behaviour we find is reminiscent of the case of one driven oscillator, weakly coupled to a set of four other oscillators (figure 6). The driven oscillator goes through a period-doubling sequence, while the other four act as bystanders in the process. The slow eigenvalue with larger phase $\varphi_{i}=\arg \left(\lambda_{i}\right)$ always moves faster, so these do not catch up. However, the fastest one can pass $\varphi=\pi$, while remaining on the circle. But when $\lambda_{1}$ meets $\lambda_{2}^{*}$, these do move away from the inner circle. This occurs always when any two multipliers $\lambda_{i}=\lambda_{j}^{*}$ $(i \neq j ; i, j \neq 0)$ meet. When the excursion is large, the outer one can cross the unit circle and give a Hopf bifurcation, but that was not observed for this $v$.
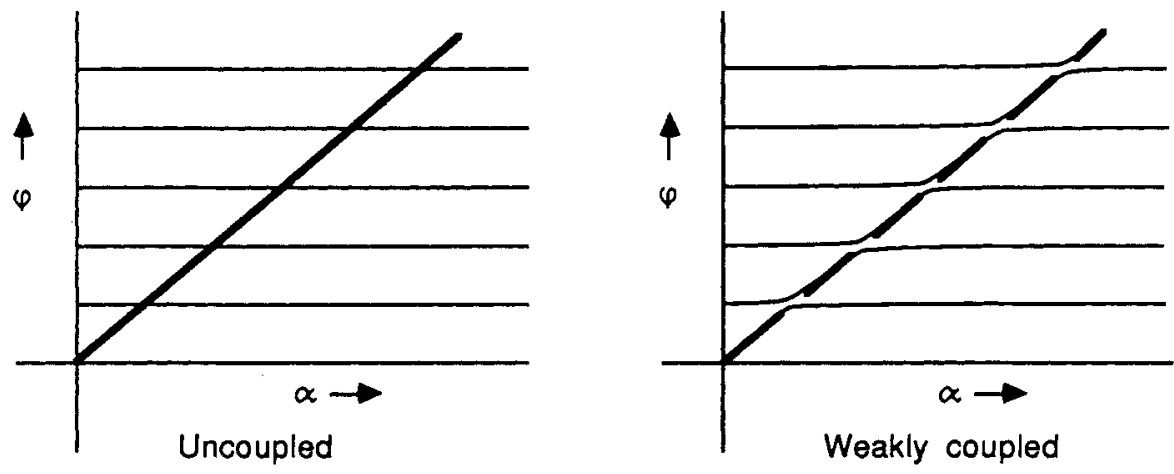

Figure 6. Behaviour of the phases of the Floquet multipliers for a set of oscillators. One of the oscillators goes through a period-doubling sequence as function of the driving parameter $\alpha$, while the others are not influenced. In the case of an uncoupled system this is trivially so, but when a weak coupling between the driven oscillator and the other ones is introduced one should expect avoided-crossing behaviour. The scale on which this avoidance takes place can be very small, depending on the actual coupling strength. 


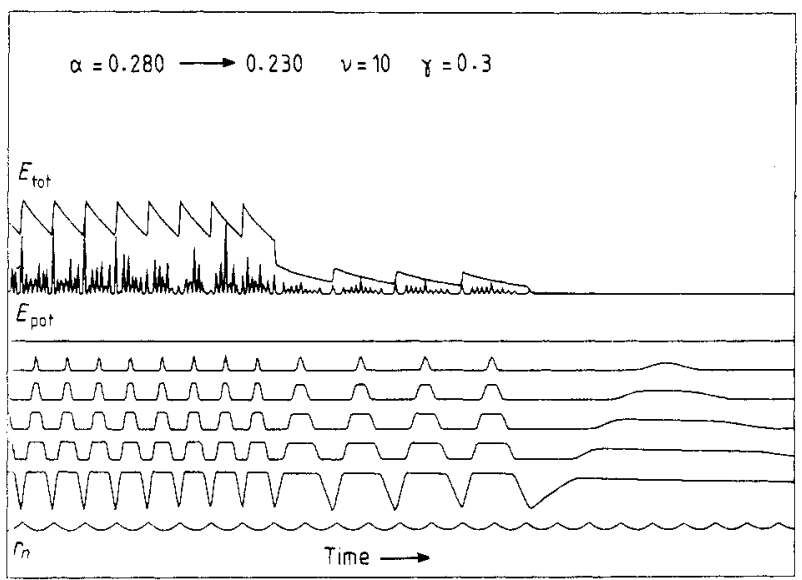

Figure 7. Decay of the single period-one density wave when the amplitude of the driving motion decreases slowly. For $\alpha$ large enough the collision between the driven end and the first particle compensates for the friction losses during one period, and the wave remains intact. When the maximal velocity of the moving wall becomes too small the single localised density wave loses momentum, and finally disappears altogether.

We have also investigated what happens at $v=10$ when we decrease $\alpha$. We could follow the stable-wave solution to $\alpha=0.280$. For a lower value there is no stable solution of the type we want to investigate, because the driving force becomes too small to compensate for the friction losses. For a single particle $(N=1)$ we have performed the same experiment. Obviously only the fast-moving pair of eigenvalues appears. For increasing $\alpha$ we find the period-doubling sequence. For decreasing $\alpha$ in the period-one orbit we find that $\varphi \rightarrow 0$, and the wave destabilises when one of the eigenvalues crosses the unit circle at $\lambda=1$. For five particles $(N=5)$ the situation is complicated by the slow vibrations. For decreasing $\alpha$ also their phase $\varphi_{i} \rightarrow 0$, and adiabatic change becomes difficult, if not impossible. Nevertheless it is quite clear from the $r-t$ plot to see how the wave destabilises when the amplitude drops below threshold (figure 7).

Our second experiment was performed at $v^{-1}=0.3$. When we track the Floquet multipliers as a function of $\alpha$ we observe two period doublings. Then one of the encounters between $\lambda_{i}$ and $\lambda_{j}^{*}$ leads to a Hopf bifurcation. However, when $\lambda_{i}$ crosses the unit circle the system does not relax to the low-periodic (ir)rational modulation of the carrier wave resulting from such a bifurcation. Instead after a rather long and very irregular transient we obtained a state in which two solitons bounce between the ends of the chain. In an earlier paper [6] we have discussed the existence of such states. Apparently we entered the basin of that orbit when we changed $\alpha$ beyond the bifurcation value.

The third systematical experiment has been performed at a short driving period, $v^{-1}=0.03$. The starting state at $\alpha=1.0$ was obtained by increasing $v$ in the way described earlier. When $v$ is varied $\lambda_{0}$ hardly changes, while the other eigenvalues scale rather trivially; the phase change is roughly proportional to the sampling period. The Floquet multiplier of the slowest vibration (smallest $\varphi$ ) goes onto the real axis, but stays within the unit circle. Increasing $\alpha$, we were able to observe the period doublings as in the case of $v=10$, and a chaotic state was found at slightly higher value of the strength parameter $\alpha=2.7$. Indeed for higher values of $v$ all orders of the period-doubling sequence occur at slightly higher values of $\alpha$, and vice versa. 


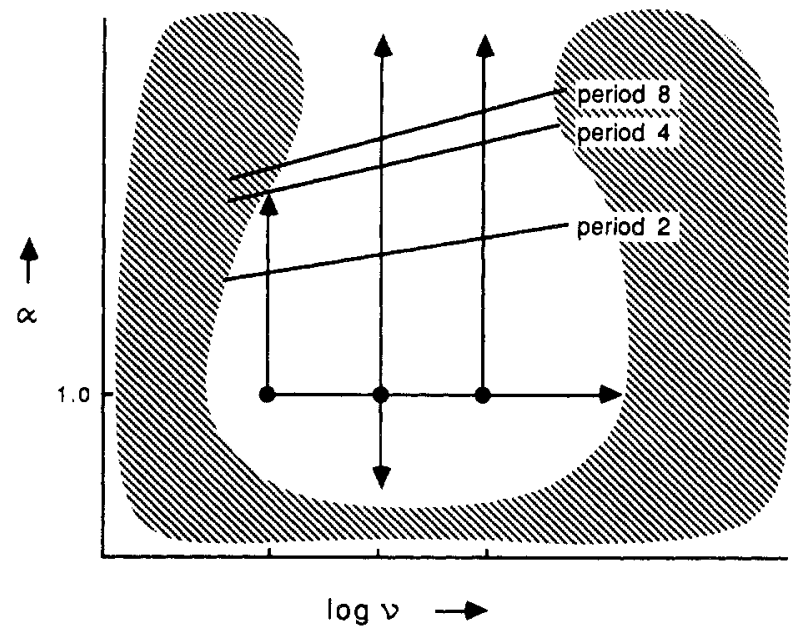

Figure 8. Sketch of the results, summarising the different experiments we have performed. In the area of the $\alpha-v$ plane in the middle of the figure there exists a localised single-density wave bouncing between the ends of the chain. For two values of $v$ we found a period doubling cascade to chaos, the consecutive period doublings appearing at slightly higher $\alpha$ for higher $v$. Outside that area the single density wave does not form a stable solution (hatched area). At the boundaries of these areas bifurcations other then period doubling occur. For low $v$ the period-doubling cascade ends when the encounter of two slow eigenvalues gives a Hopf bifurcation. For high $v$, as well as for low $\alpha$ one of the slow eigenvalues gives a destabilisation by going to +1 . The infinitely long transients in this case make the transition quite intractable.

As we said, for short driving periods we observe approximate scaling of the phases $\varphi_{1}, \ldots, \varphi_{4}$ with the period. For $v^{-1}=0.03$ we already found $\varphi_{4}=0$. When $v$ keeps rising, $\lambda_{4}$ can go outside the unit circle, possibly due to non-adiabatic effects. In that case it is not justified to calculate the Floquet multipliers over a non-closed orbit. If we fix $v$, the orbit becomes closed, and one finds all eigenvalues inside the unit circle. This is similar to the phenomenon we observed when for $v=10$ we lowered $\alpha$ below its threshold value. The appearance of these long transients inhibits further examination of the destabilisation behaviour of the wave at high driving frequencies.

In summary, we obtain a region in the $\alpha-v$ plane, as indicated in figure 8 , where there exists a localised density wave undergoing a period-doubling transition to chaos. During this period-doubling process the wave maintains its spatially coherent character (figure 4). The motion is similar to a system with only one degree of freedom (as for instance the Fermi bouncing ball [7]). The Floquet multipliers along the stable branch behave as those of a system with one dominant oscillator with a single degree of freedom, only weakly coupled to a set of other oscillators, which do not influence the period-doubling cascade. At the bounds of the region in the $\alpha-v$ plane the wave loses its stability through other than period-doubling bifurcations, and the simple picture given above breaks down.

\section{An analytical model}

Here we establish analytically a basis for the conclusions as formulated in the previous section. 
The canonical equations of the conservative chain are, after scaling of the time,

$$
v w^{\prime}=J \nabla H(w) \quad w=\left(p_{1}, r_{1}, p_{2}, \ldots, r_{N}\right) \quad H=(p, p) / 2+U(r)
$$

where $J$ is the antisymmetric matrix given in (2.6) and $U$ is the potential as in (2.1b) with $r_{0}=r_{0}(\tau, \alpha)=0$.

As a starting point to construct a model we now make the following two assumptions.

(A) The equations (4.1) have a continuous and differentiable family of periodone solutions

$$
\{\boldsymbol{v}(I, \tau), v(I)\}, I=\oint\left(\boldsymbol{p}, \boldsymbol{r}^{\prime}\right) \mathrm{d} \tau
$$

which represents a bouncing solitary wave with frequency $v(I)$.

Below we discuss arguments in favour of this assumption. For our purpose it is sufficient to assume that existence of this family on some interval $I_{\min }<I<I_{\max }$.

To formulate our second assumption observe that the family $v(I, \tau)$ defines a two-dimensional surface in phase space

$$
V=\left\{\boldsymbol{v}(I, \tau) \mid I_{\min }<I<I_{\max }, 0 \leqslant \tau \leqslant 1\right\} .
$$

Now the observation of $\S 3$ that the wave maintains its solitary wave character during the period-doubling cascade justifies the following tentative conclusion. i.e.

(B) The trajectory of the damped driven system remains 'near' the surface $V$,

$$
w(t)=\boldsymbol{v}(I(t), \tau(t))+z(t)
$$

where $z$ remains small.

Some comments are in order here. First note that (cf (4.2)) $I$ and $\tau$ can be interpreted as action angle variables on the surface $V$. Correspondingly an orbit in the neighbourhood of $V$ can be written as in (4.4a) with $I$ and $\tau$ depending on $t$ in an a priori unknown way. Secondly, $z$ is required to be ' $J$-orthogonal' to $V$ for all time, i.e.

$$
\left(v_{l}, J z\right)=\left(v^{\prime}, J z\right)=0 \quad \text { with } \quad v_{I}=\partial v / \partial I
$$

The effect of this requirement is twofold: for a given trajectory of the chain it makes the splitting (4.4a) unique and, related to that, it allows us to separate the dynamical equations for $I$ and $\tau$ on the one hand, and for $z$ on the other.

First, however, consider assumption A. We give some arguments in favour of the existence of the family $v(I, \tau)$.

(i) The family exists for the Toda chain. In that case, for low energy (action), it is the ordinary standing wave with longest possible wavelength and for increasing action it sharpens up to a bouncing solitary wave [8].

(ii) It is well known that such a standing wave bifurcates from the equilibrium in the Lennard-Jones chain (cf also [9]). Typically such a bifurcating branch can be continued [10]. Although a priori not much is known about its properties, we mention two points which indicate that the wave here also sharpens to a bouncing solitary wave. Firstly, for a Lennard-Jones ring, there is a family which represents for low energy the travelling wave with longest wavelength which develops to a sharp solitary wave running around in the chain [11]. This sharpening is a consequence of the growing steepness of the interaction potential for small distance between the particles. This becomes clear from the fact that this travelling wave is a critical point of a variational problem (roughly speaking, it is a maximum of the time-averaged 
potential energy for constant kinetic energy). Secondly, the bifurcating branch of the present case can also be formulated as a critical point of a variational problem. Here the analysis is much more complicated since the critical point is a saddle but, nevertheless, if the solution exists it is also expected to sharpen up for increasing energy. Work on this point is in progress.

Now consider the observation B. We derive equations of motion for $I, \tau$ and $z$ which are valid to first order in $z$ in the neighbourhood of $V$. To this end we define an appropriate coordinate system for $z$ on the basis of the approximate linearised equation

$$
v y^{\prime}=J A(\tau) y \quad A(\tau)=\mathbf{d} \nabla H(v(\tau))
$$

for small variations $y$ about a solution $w=\boldsymbol{v}(I, \tau)$ of (4.1). This is a linear equation with periodic coefficients whose theoretical treatment is standard (Floquet theory, cf [5]). This theory yields the following lemma.

\section{Lemma.}

(i) If the time-one map of (4.5) has no negative real eigenvalues, then there is a real symplectic matrix $S(I, \tau)$ with period one, and a real symmetric matrix $K(I)$, such that the coordinate transformation $y=S x$ transforms (4.5) to

$$
v x^{\prime}=J K x \text {. }
$$

(ii) $S$ can be chosen in such a way that

$$
S=\left[\boldsymbol{v}_{l}, \boldsymbol{v}^{\prime}, \ldots\right] \quad \text { and } \quad K=K_{0} \oplus K_{1}
$$

where $K_{0}=\operatorname{diag}\left(v_{I} / v, 0\right)$ and $K_{1}$ is a $2(N-1) \times 2(N-1)$ matrix.

The first two columns of $S, \boldsymbol{v}_{I}$ and $\boldsymbol{v}^{\prime}$, are tangent to $V$. The other columns are $J$-orthogonal to these tangent vectors, since $S$ is symplectic,

$$
S^{\mathrm{T}} J S=J .
$$

Now write $w$ in the neighbourhood of the surface $V$ as

$$
w=v(I, \tau)+S(I, \tau) \zeta \quad \zeta=\operatorname{col}\left(0,0, \alpha_{2}, \beta_{2}, \ldots, \alpha_{N}, \beta_{N}\right)
$$

which defines a coordinate transformation $w=\boldsymbol{w}(I, \tau, \zeta)$. The vectorial derivative of $w$ is

$$
\mathbf{d} w=S+G \quad G=\left[S_{I} \xi, S^{\prime} \xi, \ldots\right] .
$$

Observe that $G=\mathrm{O}(\zeta)$. Transforming the equations of motion to the new variables we also need

$$
\mathbf{d} w^{-1}=\left(1+S^{-1} G\right)^{-1} S^{-1}=S^{-1}-T+\mathrm{O}\left(\zeta^{2}\right) \quad T=S^{-1} G S^{-1} .
$$

For the interpretation of the approximate equations below it is important to observe that the terms $G$ and $T$ are linear in $\zeta$ and that they originate from the variation of the coordinate system along $V$ ( $\mathrm{cf}(4.10 a)$ ).

To obtain the equations in terms of the new coordinates we write the equations of motion of the driven and damped system as

$$
(\mathrm{d} / \mathrm{d} t) w=J \nabla H(w)+J \nabla F(w, t)-\lambda P_{p} w .
$$


Here $P_{p}$ is the projection operator on the momentum part of the phase space

$$
P_{p}=\operatorname{diag}(1,0,1,0,1, \ldots, 0)
$$

and the time-dependent term for our chain is given by (cf $(2.1 b)$ )

$$
F=V\left(r_{1}-r_{0}(t, \alpha)\right)-V\left(r_{1}-r_{0}(t, 0)\right) \text {. }
$$

To obtain the equations in new variables $\xi$

$$
\xi=\operatorname{col}\left(I, \tau, \zeta_{1}, \zeta_{2}, \ldots, \zeta_{2 N-2}\right)
$$

we make use of

$$
(\mathrm{d} / \mathrm{d} t) \xi=\mathbf{d} w^{-1}(\mathrm{~d} / \mathrm{d} t) w
$$

so that

$$
(\mathrm{d} / \mathrm{d} t) \xi=\mathbf{d} w^{-1} J\{\nabla H+\nabla F\}-\lambda \mathbf{d} w^{-1} P_{p}(v(I, \tau)+S \zeta) .
$$

Evaluation of the conservative part is straightforward (although a little tricky). It yields

$P_{0} \mathbf{d} w^{-1} J \nabla H=(0, v(I))^{\mathrm{T}}+\mathrm{O}(\zeta) \quad P_{1} \mathbf{d} w^{-1} J \nabla H=J K_{1}+\mathrm{O}\left(\zeta^{2}\right)$

$P_{i}$ denoting the projection operators

$$
P_{0}=\operatorname{diag}(1,1,0, \ldots, 0) \quad P_{1}=\operatorname{diag}(0,0,1, \ldots, 1) .
$$

Then we obtain from (4.14)

$$
(\mathrm{d} / \mathrm{d} t)(I, \tau)^{\mathrm{T}}=(0, v(I))^{\mathrm{T}}+P_{0} S^{-1} J(\nabla F)(v(I, \tau) ; t)-\lambda P_{0} S^{-1} P_{p} v(I, \tau)+\mathrm{O}(\zeta)
$$

In the first two terms on the right-hand side, one recognises a driven nonlinear Hamiltonian oscillator. The third term represents a dissipation which causes a decrease of the action as will be shown now (cf (4.20)). To evaluate this term we use

$$
S^{-1}=-J S^{\mathrm{T}} J
$$

which follows from (4.7), to obtain

$$
\begin{aligned}
P_{0} S^{-1} P_{p} v(I, \tau) & =-P_{0} J S^{\mathrm{T}} J \boldsymbol{p}=-P_{0} J\left(\left(\boldsymbol{v}_{I}, J \boldsymbol{p}\right),\left(\boldsymbol{v}^{\prime}, J \boldsymbol{p}\right), \ldots\right)^{\mathrm{T}} \\
& =\left(\left(\mathbf{v}^{\prime}, \mathrm{J} \mathbf{p}\right),-\left(\mathbf{v}_{\mathrm{I}}, \mathrm{J} \mathbf{p}\right), 0, \ldots, 0\right)
\end{aligned}
$$

with $p=P_{p} v$. With

$$
\boldsymbol{v}^{\prime}=\left(p_{1}^{\prime}, r_{1}^{\prime}, p_{2}^{\prime}, \ldots\right) \quad \text { and } \quad \boldsymbol{p}=\left(v r_{1}^{\prime}, 0, v r_{2}^{\prime}, 0, \ldots\right)
$$

we finally obtain

$$
P_{0} S^{-1} \boldsymbol{p}=\left(v^{-1}(\boldsymbol{p}, \boldsymbol{p}),\left(v_{1}, J \boldsymbol{p}\right), 0, \ldots\right)^{\mathrm{T}} .
$$

Observe in particular that the first term is non-negative, so that it contributes to a decrease of the action.

The equation for $\zeta$ is

$$
\begin{aligned}
(\mathrm{d} / \mathrm{d} t) \zeta=P_{1}[ & J K_{1} \zeta+S^{-1} J \nabla F(\boldsymbol{v}(I, \tau) ; t) \\
& -T J \nabla F(\boldsymbol{v}(I, \tau) ; t)+S^{-1} J \mathbf{d} \nabla F(\boldsymbol{v}(I, \tau) ; t) S \zeta \\
& \left.-\lambda\left\{S^{-1}-T\right\} \boldsymbol{p}(I, \tau)-\lambda S^{-1} P_{p} S \zeta+\mathrm{O}\left(\zeta^{2}\right)\right] .
\end{aligned}
$$

The first term on the right represents a set of linear oscillators. The second term represents an inhomogeneous external forcing, namely the component of the 
external force in the $\zeta$ direction. The third and fourth term have the same origin but are homogeneous (recall that $T=\mathrm{O}(\zeta)$ ).

The first term with $\lambda$, proportional to $p$, acts also as a forcing. The last term is the 'ordinary' dissipation (with dissipation constant a function of $I$ and $\tau$ ).

Summarising, equations (4.16) and (4.21) justify the following conclusions.

(i) If the trajectory of the system remains near the surface $V$, the main part of the motion is described by (4.16), which represents a driven damped nonlinear oscillator in one degree of freedom. The characteristic properties of this oscillator such as frequency and dissipation rate, are given by the family $v(I, \tau)$.

(ii) Such trajectories are to be expected if the orbits of the inhomogeneously driven linear ' $\zeta$ ' oscillator (4.21) has bounded orbits which are sufficiently small. Whether this is true or not depends on the external forcing and on properties of the family $v(I, \tau)$. The (computational) experimentalist has the former in hand. The latter cannot be influenced. The only freedom one has is to choose a driving frequency in such a way that one selects a part of the surface $V$ where the $\zeta$ motion remains sufficiently small.

Thus we conclude that the period-doubling behaviour described in $\$ 3$ can be explained by the assumptions $\mathrm{A}$ and $\mathrm{B}$, provided that the external forcing is chosen suitably and that the family $v(I, \tau)$ has the right geometrical properties.

\section{Acknowledgment}

The authors thank $\mathrm{E}$ van Groesen for illuminating discussions.

\section{Appendix}

Here we describe the discretisation of the continuous equations $(2.1 a)$

$$
r^{\prime \prime}=-v^{-2} \operatorname{grad} U(r ; \tau)-\gamma r^{\prime} \quad r \in R^{N} \text {. }
$$

Choose an interval $\Delta \tau=1 / K$, where $K$ is an integer, which equals 400 in the calculations in this paper. An orbit $r(\tau)$ of the continuous system is now replaced by a discrete orbit $\{\ldots, r(i), r(i+1), \ldots\}$ with $r(i)=r(i \Delta \tau)$. To obtain a secondorder difference equation that describes this discrete orbit approximately, observe that (A1) is a stationary point of the action functional

$$
A_{\mathrm{c}}=\int \mathrm{d} \tau \mathrm{e}^{\gamma \tau}\left(\left(r^{\prime}, r^{\prime}\right) / 2-v^{-2} U(r ; \tau)\right)
$$

We now replace the action $A_{\mathrm{c}}$ by

$A_{\mathrm{d}}=\sum_{j} \mathrm{e}^{j \gamma \Delta \mathrm{r}}\left[(r(j)-r(j-1), r(j)-r(j-1)) / 2-v^{-2} \Delta \tau^{2} U(r(j) ; j \Delta \tau)\right]$.

To obtain a second-order difference equation take the summation boundaries $i \leqslant j \leqslant i+1$. Then vary the resulting action with the endpoints $r(i-1)$ and $r(i+1)$ fixed, i.e. differentiate with respect to $r(i)$, to find

$$
\begin{aligned}
& r(i)-r(i-1)-\rho^{-1}(r(i+1)-r(i))=\mu^{2} \operatorname{grad} U(r(i) ; i \Delta \tau) \\
& \rho^{-1}=\mathrm{e}^{\gamma \Delta \tau} \quad \mu^{2}=(\Delta \tau / v)^{2} .
\end{aligned}
$$


Observe that this last system is a well defined dynamical system in its own right. With the continuous equations it has in common that the Jacobians are a constant and reduce volume in phase space at the same rate. We do not bother very much about possible discrepancies between the solutions of the continuous and the discrete system. In practice one can choose $\Delta \tau$ sufficiently small such that the orbits of the latter can readily be interpreted as orbits of the former.

\section{References}

[1] Bishop A R, Grüner G and Nicolaenko B (eds) 1986 Proc. Conf. on Spatio-Temporal Coherence and Chaos in Physical Systems (Los Alamos 1986) Physica 23D

[2] Adachihara H, McLaughlin D W, Moloney J V and Newell A C 1988 J. Math. Phys. 29 63-85

[3] Geist K and Lauterborn W 1987 Physica 31D 103-16

Satija I I, Bishop A R and Fesser K 1985 Phys Lett. 112A 183-7

Fesser K, McLaughlin D W, Bishop A R and Holian B L 1985 Phys Rev A 2728-731

[4] Bishop A R, Forest M G, McLaughlin D W and Overman E A II 1986 Physica 23D 293-328

Bishop A R 1985 Dynamical Problems in Soliton Systems (Proc. 7th Kyoto Summer Institute) ed S Takeno (Berlin: Springer) pp 250-7

Bishop A R, Fesser K, Lomdahl P S and Trullinger S E 1983 Physica 7D 259-79

[5] Yakubovich V A and Starzhinskii V M 1975 Linear Differential Equations with Periodic Coefficients vol 1 (New York: Wiley)

[6] Valkering T P and van Opheusden J H J 1986 Physica 23D 381-90

[7] Lichtenberg A $J$ and Lieberman M A 1983 Regular and Stochastic Motion (Berlin: Springer)

[8] Ferguson W E, Flaschka H and McLaughlin D W 1982 J. Comput. Phys. 45 157-209.

[9] van Gils S A and Valkering T P 1986 Japan J. Appl. Math. 3 207-27

[10] Rabinovitz P H 1971 J. Funct. Anal. 7 487-513

[11] Valkering T P 1982 Cel. Mech. 28 119-31

Valkering T P and van Opheusden J H J 1989 Proc. Conf. on Nonlinear Dynamics, Bologna, 1988 ed G Turchetti (Singapore: World Scientific) 